\title{
Low-lying eigenmodes and meson propagator symmetries
}

\author{
C. B. Lang* \\ Institute of Physics, University of Graz, A-8010 Graz, Austria
}

(Received 29 March 2018; published 20 June 2018)

\begin{abstract}
In situations where the low-lying eigenmodes of the Dirac operator are suppressed one observed degeneracies of some meson masses. Based on these results a hidden symmetry was conjectured, which is not a symmetry of the Lagrangian but emerges in the quantization process. We show here how the difference between classes of meson propagators is governed by the low modes and shrinks when they disappear.
\end{abstract}

DOI: 10.1103/PhysRevD.97.114510

\section{MOTIVATION}

Recently it was found that in certain situations a symmetry emerges that relates vector and scalar meson propagators but that is no symmetry of the action. That symmetry was observed in lattice QCD when low-lying eigenmodes of the Dirac operator are suppressed either artificially by removing the eigenmodes from the quenched quark propagators [1-5] or naturally in the high temperature phase $[6,7]$ either due to a gap ${ }^{1}$ or because another rapid decrease toward zero eigenvalues. The symmetry group was called CS (chiral-spin) and has been suggested $[12,13]$ to be $S U(4) \supset S U(2)_{L} \times S U(2)_{R} \times U(1)_{A}$ mixing the $u$ - and $d$-quarks of a given chirality and also the leftand right-handed components.

Here we provide results to elucidate the observed symmetry. We find explicitly in an analytic calculation that some propagator identities emerge if the low-lying eigenmodes of the Dirac operator are suppressed. We show that in that case propagators of different mesons become degenerate giving rise to the CS symmetry in part. For the other part of the symmetry further conditions have to be met.

Consider the eigenvalues of the Hermitian Dirac operator. It is well known that the difference of the susceptibilities of, e.g., the propagator of the isovector scalar meson operator and of the isovector pseudoscalar operator are weighted by an eigenvalues density factor (on top of the generic eigenvalue density), that approaches a delta function in the zero mass limit. The approach is similar to the derivation of the Banks-Casher relation for the quark condensate [14]. The difference between the scalar and

\footnotetext{
*christian.lang@uni-graz.at

${ }^{1}$ The existence of a gap above the finite temperature transition in lattice QCD is disputed [8-11].

Published by the American Physical Society under the terms of the Creative Commons Attribution 4.0 International license. Further distribution of this work must maintain attribution to the author(s) and the published article's title, journal citation, and DOI. Funded by SCOAP ${ }^{3}$.
}

pseudoscalar propagators and susceptibilities has been intensely studied earlier [15-17]. We show here that this property applies to a large set of (scalar, pseudoscalar, vector and axial vector) meson propagator pairs and discuss the conditions for the CS symmetry.

\section{NOTATION}

\section{A. Dirac operator}

We work in Euclidean space-time continuum and will briefly remind on the notation. The tool will be the spectral representation of the Dirac quark and the meson propagator. As there are sums over all (an infinite number of) eigenmodes we need some regularization (e.g., a finite volume lattice) and for this we rely on Fujikawa's approach [18-20], which we assume implicitly but will omit the actual derivation.

We choose hermitian $\gamma$-matrices $\gamma_{\mu}^{\dagger}=\gamma_{\mu}$ and $\left[\gamma_{\mu}, \gamma_{\nu}\right]_{+}=$ $2 \delta_{\mu \nu}$. The fermions are Grassmann fields and the Dirac action

$$
\int d^{4} x \bar{\psi}\left(\mathrm{i} \gamma_{\mu} D_{\mu}+\mathrm{i} m\right) \psi
$$

is real. The massless Euclidean Dirac operator $D \equiv \mathrm{i} \gamma_{\mu} D_{\mu}$ is hermitian with the eigensystem

$$
D \psi_{x, a}^{(n)}=\eta_{n} \psi_{x, a}^{(n)}
$$

The dimension of the eigenvectors is $n_{D} n_{c} n_{f}$ (Dirac, color, flavor) at each point $x \in \mathbb{R}^{4}$ and there are $n_{D} n_{c} n_{f}$ eigenvectors as functions of $x$. Only the Dirac index $a$ is kept explicitly, the color- and flavor indices are implicit. The non-zero eigenvalues come in pairs as can be seen by multiplying with $\gamma_{5}$ :

$$
\begin{aligned}
\gamma_{5} D \psi^{(n)} & =\eta_{n} \gamma_{5} \psi^{(n)} \\
\rightarrow D\left(\gamma_{5} \psi^{(n)}\right) & =-\eta_{n}\left(\gamma_{5} \psi^{(n)}\right) .
\end{aligned}
$$

We use the notation $\eta_{n} \equiv \eta_{n}$ with $\eta_{-n}=-\eta_{n}$ and $\psi^{(-n)}=$ $\gamma_{5} \psi^{(n)}$. The eigenvalues are real and the eigenvectors form an orthonormal basis 


$$
\sum_{a} \int d^{4} x \psi_{x a}^{(n) \dagger} \psi_{x a}^{(k)}=\delta_{n k}
$$

We formally regularize by point-splitting such that the Dirac operator becomes a matrix,

$$
D_{x a \mid y b} \psi_{y b}^{(n)}=\eta_{n} \psi_{x a}^{(n)} .
$$

The indices for color and flavor are implicit. For simplicity we assume mass degenerate fermions. The Dirac operator has the spectral representation

$$
D_{x a \mid y b}+\mathrm{i} m \delta_{x a \mid y b}=\sum_{n}\left(\eta_{n}+\mathrm{i} m\right) \psi_{x a}^{(n)} \psi_{y b}^{(n) \dagger} .
$$

The fermion propagator then has the representation

$$
(D+\mathrm{i} m)_{x a \mid y b}^{-1}=\sum_{n} \frac{1}{\eta_{n}+\mathrm{i} m} \psi_{x a}^{(n)} \psi_{y b}^{(n) \dagger} .
$$

We assume that there are no exact zero modes with $\gamma_{5} \psi= \pm \psi$ either because we are in the topological sector zero or because they have been removed. Anyhow they are suppressed in the thermodynamic limit.

\section{B. Chiral symmetry}

We define chiral symmetry as the invariance of the massless Dirac operator. The transformation is

$$
\begin{array}{ll}
\psi(x)^{\prime}=\mathrm{e}^{\mathrm{i} \alpha \gamma_{5} \mathbf{1}_{f}} \psi(x), & \bar{\psi}(x)^{\prime}=\bar{\psi}(x) \mathrm{e}^{\mathrm{i} \alpha \gamma_{5} \mathbf{1}_{f}}, \\
\psi(x)^{\prime}=\mathrm{e}^{\mathrm{i} \alpha \gamma_{5} \tau_{i}} \psi(x), & \bar{\psi}(x)^{\prime}=\bar{\psi}(x) \mathrm{e}^{\mathrm{i} \alpha \gamma_{5} \tau_{i}},
\end{array}
$$

where $\tau_{i}$ are the generators of $S U(2)_{\text {flavor }}$ and $\mathbf{1}_{f}$ the unit matrix in flavor space. The kinetic term of the action is invariant, e.g.,

$$
\bar{\psi} \gamma_{\mu} \psi \rightarrow \bar{\psi} \mathrm{e}^{\mathrm{i} \alpha \gamma_{5}} \gamma_{\mu} \mathrm{e}^{\mathrm{i} \alpha \gamma_{5}} \psi=\bar{\psi} \gamma_{\mu} \psi
$$

The chiral transformation commutes with the Euclidean Lorentz transformations $O(4)$.

For the discussion it will be useful to split the four-Diraccomponents of the eigenvectors into a pair,

$$
\psi^{(n)}=\psi_{R}^{(n)}+\psi_{L}^{(n)}
$$

with

$$
\psi_{R}^{(n)}=\frac{1}{2}\left(\mathbf{1}+\gamma_{5}\right) \psi, \quad \psi_{L}^{(n)}=\frac{1}{2}\left(\mathbf{1}-\gamma_{5}\right) \psi .
$$

We choose a chiral basis for the Dirac matrices with $\gamma_{5}=$ $\operatorname{diag}(1,1,-1,-1)$ such that

$$
\psi_{R}^{(n)}=\left(\begin{array}{c}
R^{(n)} \\
0
\end{array}\right), \quad \psi_{L}^{(n)}=\left(\begin{array}{c}
0 \\
L^{(n)}
\end{array}\right),
$$

and $R, L$ having two components.

\section{CS symmetry}

The (Hermitian) $S U(2)_{\mathrm{CS}}$ (shorter: CS for "chiral spin") algebra [12] generators are

$$
\mathrm{CS}_{k}: T \in\left\{\gamma_{k}, \mathrm{i} \gamma_{k} \gamma_{5}, \gamma_{5}\right\}, \quad k=1,2,3,4 .
$$

We define the transformation guided by the Minkowski version (i.e., that $\bar{\psi}$ should transform like $\psi^{\dagger} \gamma_{4}$.)

$$
\psi \rightarrow \psi^{\prime}=\mathrm{e}^{\mathrm{i} \alpha T} \psi, \quad \bar{\psi} \rightarrow \bar{\psi}^{\prime}=\bar{\psi} \gamma_{4} \mathrm{e}^{-\mathrm{i} \alpha T^{\dagger}} \gamma_{4}
$$

Then quark-antiquark bilinears transform like

$$
\bar{\psi} \mathcal{O} \psi \rightarrow \bar{\psi}\left[\gamma_{4} \mathrm{e}^{-\mathrm{i} \alpha T_{a}^{\dagger}} \gamma_{4}\right] \mathcal{O} \mathrm{e}^{\mathrm{i} \alpha T_{b}} \psi
$$

where $T_{a}, T_{b}$ are generators of the algebra CS. Each operator this way is an element of a multiplet. E.g., a $\mathrm{CS}_{1}$ multiplet might be $\left(\gamma_{2}, \mathrm{i} \gamma_{2} \gamma_{5}, \mathrm{i} \gamma_{4} \gamma_{3}, \mathrm{i} \gamma_{2} \gamma_{1}\right)$ which corresponds to $\rho, \omega, a_{1}, f_{1}, b_{1}, h_{1}$ for isovectors and isoscalars.

For $T=\gamma_{5}$ this is the transformation of chiral symmetry (8). Only $\gamma_{5}$ leaves the kinetic term invariant. It turns out that only $\gamma_{5}$ is anomalous giving a factor for the Grassmann path integral integration measure. All CS transformations leave the chemical potential term $\bar{\psi} \gamma_{4} \psi$ invariant. In $[12,13]$ the embedding of $S U(2)_{\mathrm{CS}} \times S U(2)_{f} \subset S U\left(2 n_{f}\right)$ was suggested. In $S U(4)$ the vector mesons form a 15-plet $\left(\rho, \rho^{\prime}, b_{1}, a_{1} \cdot h_{1}, \omega, \omega^{\prime}\right)$ and a singlet $\left(f_{1}\right)$.

The CS transformations as a whole are no symmetry of the Dirac action. However, it has been observed, that CS is a symmetry of certain meson and baryon masses, if the lowlying (quasizero) modes are absent [3-5,21]. At zero temperature, with artificial removal of low-lying modes in the valence sector, confinement seems to persist. Above the chiral temperature the zero modes are suppressed naturally. There are indications that some form of confinement persists as well [7].

The chromo-electric observables $\bar{\psi} \gamma_{4} \psi$ are symmetric under CS, the kinetic term of the action and the chromomagnetic terms $\bar{\psi} \gamma_{k} \psi(k=1,2,3)$ are not. Removing the near-zero modes apparently restores the symmetry such that the influence of chromo-magnetism shrinks or disappears. One might conclude that confinement has its origin in the chromo-electric sector, which is symmetric under CS always [22].

\section{MESON PROPAGATORS}

We restrict ourselves to two mass degenerate quark flavors $u$ and $d$. As already mentioned, we neglect exact zero modes, either because we are in that topological sector or because they have been removed.

We study the propagators for mesons of type $\bar{\Psi}(\vec{\tau} \otimes \Gamma) \Psi$ and $\bar{\Psi}\left(\mathbf{1}_{f} \otimes \Gamma\right) \Psi$. The $\Gamma$ are listed in Table I; the choice has been motivated by the discussion of the CS symmetry in Ref. [12]. We emphasize that our results are for the propagators themselves, not just the masses. 
TABLE I. We list the sink and source operator kernels. We also give sign factors $s_{5}$ defined by $\Gamma \gamma_{5}=s_{5} \gamma_{5} \Gamma$.

\begin{tabular}{lcccr}
\hline \hline$\Gamma_{\text {src }}$ & $\Gamma_{\text {snk }}$ & $\mathrm{i} \Gamma_{\text {src }} \gamma_{5}$ & $\mathrm{i} \gamma_{5} \Gamma_{\text {snk }}$ & $s_{5}$ \\
\hline $\mathbf{1}$ & $\mathbf{1}$ & $\mathrm{i} \gamma_{5}$ & $\mathrm{i} \gamma_{5}$ & 1 \\
$\gamma_{k}$ & $\gamma_{k}$ & $\mathrm{i} \gamma_{k} \gamma_{5}$ & $-\mathrm{i} \gamma_{k} \gamma_{5}$ & -1 \\
$\gamma_{4}$ & $\gamma_{4}$ & $\mathrm{i} \gamma_{4} \gamma_{5}$ & $-\mathrm{i} \gamma_{4} \gamma_{5}$ & -1 \\
$\gamma_{k} \gamma_{j}$ & $-\gamma_{k} \gamma_{j}$ & $\mathrm{i} \gamma_{k} \gamma_{j} \gamma_{5}$ & $-\mathrm{i} \gamma_{k} \gamma_{j} \gamma_{5}$ & 1 \\
$\gamma_{k} \gamma_{4}$ & $-\gamma_{k} \gamma_{4}$ & $\mathrm{i} \gamma_{k} \gamma_{4} \gamma_{5}$ & $-\mathrm{i} \gamma_{k} \gamma_{4} \gamma_{5}$ & 1 \\
\hline \hline
\end{tabular}

For the isotriplet we introduce the connected propagator for a given background gauge field $A$,

$$
\begin{aligned}
P_{c}(\Gamma, x, y) & =-\left[\bar{u}_{x a} \Gamma_{a b}^{\mathrm{snk}} d_{x b} \bar{d}_{y c} \Gamma_{c d}^{\mathrm{src}} u_{y d}\right]_{A} \\
& =\operatorname{tr}_{\text {Dirac }}\left[D_{u_{y d} \mid x a}^{-1} \Gamma_{a b}^{\mathrm{snk}} D_{d_{x b} \mid y c}^{-1} \Gamma_{c d}^{\mathrm{src}}\right]
\end{aligned}
$$

where $[\ldots]_{A}$ indicates Grassmann integration in an external field $A$. We relate source and sink by $\Gamma_{\mathrm{snk}}=\Gamma_{\mathrm{src}}^{\dagger} \equiv s_{\Gamma} \Gamma_{\text {src }}$, see Table I. For degenerate quark masses $D_{u}^{-1}=D_{d}^{-1}$. With the spectral representation for $D^{-1}$ the meson propagator becomes

$$
\begin{aligned}
P_{c}(\Gamma, x, y)= & s_{\Gamma} \sum_{n, k} \frac{1}{\left(\eta_{n}+\mathrm{i} m\right)\left(\eta_{k}+\mathrm{i} m\right)} \\
& \times \psi_{y d}^{(n)} \psi_{x a}^{(n) \dagger} \Gamma_{a b} \psi_{x b}^{(k)} \psi_{y c}^{(k) \dagger} \Gamma_{c d} \\
= & s_{\Gamma} \sum_{n, k} \frac{1}{\left(\eta_{n}+\mathrm{i} m\right)\left(\eta_{k}+\mathrm{i} m\right)} \\
& \times\left(\psi_{x a}^{(n) \dagger} \Gamma_{a b} \psi_{x b}^{(k)}\right)\left(\psi_{y c}^{(k) \dagger} \Gamma_{c d} \psi_{y d}^{(n)}\right) .
\end{aligned}
$$

The isoscalar propagators have also disconnected contributions proportional to

$$
P_{d}(\Gamma, x)=s_{\Gamma} \operatorname{tr}_{\text {Dirac }}\left[D_{u}^{-1} x b \mid x a \Gamma_{a b}\right] \operatorname{tr}_{\text {Dirac }}\left[D_{d y d \mid y c}^{-1} \Gamma_{c d}\right]
$$

Like other expressions used here, this has to be regularized (e.g., by lattice regularization) and there are standard tools to do this (e.g., [18-20]). The results and conclusions presented here are not affected.

We will find useful the identities

$$
\psi_{x a}^{(-n) \dagger} \Gamma_{a b} \psi_{x b}^{(-k)}=\psi_{x a}^{(n) \dagger}\left(\gamma_{5} \Gamma \gamma_{5}\right)_{a b} \psi_{x b}^{(k)}=s_{5} \psi_{x a}^{(n) \dagger} \Gamma_{a b} \psi_{x b}^{(k)}
$$

$\psi_{x a}^{(-n) \dagger} \Gamma_{a b} \psi_{x b}^{(k)}=\psi_{x a}^{(n) \dagger}\left(\gamma_{5} \Gamma\right)_{a b} \psi_{x b}^{(k)}=s_{5} \psi_{x, a}^{(n) \dagger} \Gamma_{a b} \psi_{x, b}^{(-k)}$,

where $\gamma_{5} \Gamma \gamma_{5}=s_{5} \Gamma$ (see Table I).

The difference between two meson propagators depends on

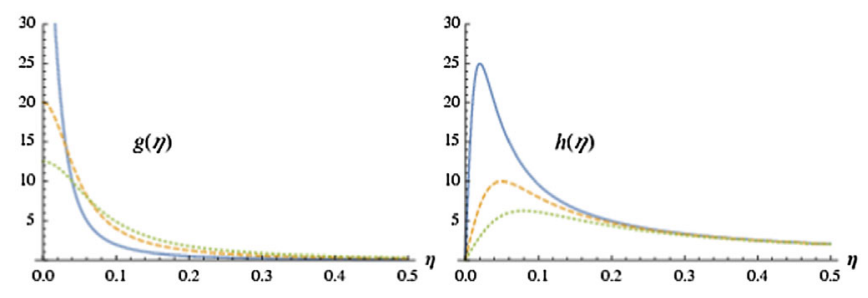

FIG. 1. The weight functions $g(\eta)$ and $h(\eta)$ from (22) for typical values $m$ equal to 0.02 (full), 0.06 (dashed) and 0.08 (dotted).

(i) the generic distribution density of eigenvalues $\rho(m, \eta)$ (which depends on the gauge configuration and the Dirac operator),

(ii) the values of the overlap matrix elements $\psi^{\dagger} \Gamma \psi$ (which are bounded from above due to the orthogonality and normalization of the eigenvectors), and

(iii) a weight function discussed below.

The generic distribution of eigenvalues $\rho(m, \eta)$ is needed only for the small eigenvalues, where it vanishes fast enough or there is even a gap, the relevant cases of this study. The bulk behavior is inconspicuous [23].

We focus here on the third factor. As derived in the Appendixes the functions (see Fig. 1)

$g(m, \eta) \equiv \frac{m}{m^{2}+\eta^{2}}, \quad h(m, \eta) \equiv \frac{\eta}{m^{2}+\eta^{2}}, \quad(\eta>0)$

turn up in the sums over eigenvalues in the next section. They are essential for the argumentation. Both functions give large weight to contributions from small $\eta$. The function $g$ is peaked at $\eta=0$ and approaches $(\pi / 2) \delta(\eta)$ for small masses $m \rightarrow 0$; for large $\eta$ it falls like $1 / \eta^{2}$. Propagator differences weighted by $g$ vanish for small $m$ if there is a gap in the density $\rho(m, \eta)$ at low-lying eigenmodes, i.e., if there are no eigenvalues below some value, or if the density vanishes fast enough for $\eta \rightarrow 0$. Propagators that differ only by terms proportional to $g$ will be called $g$-equivalent.

The function $h$ is peaked at $\eta=m$ and falls like $1 / \eta$ for large $\eta$. Compared to $g$ this behavior may not suppress the higher modes enough, depending on the Dirac structure. Propagators that differ also by terms proportional to $h$ will be called $h$-equivalent. For these the existence of a gap at low eigenvalues is not sufficient to obtain propagator agreement and more conditions have to be met.

In the next section we discuss the main results. The full derivations can be found in the Appendixes. The resulting equivalences between the meson propagators are listed in Table II and shown in Figs. 2 and 3.

\section{A. g-equivalent mesons}

\section{1. $\Gamma v s \mathrm{i} \Gamma \gamma_{5}$}

For $\Gamma \in\left\{\mathbf{1}, \gamma_{k}, \gamma_{4}, \gamma_{k} \gamma_{j}, \gamma_{k} \gamma_{4}\right\}$ the difference between meson isovector propagators is 
TABLE II. Related meson propagators; For $g$-equivalent propagators the differences vanish in the massless limit if there are no low-lying modes. Further assumptions are necessary for $h$-equivalence.

\begin{aligned} \hline \hline$g$-equivalent meson propagators \\ \hline$\tau^{a} \otimes \mathbf{1} \leftrightarrow \tau^{a} \otimes \gamma_{5} \\ \tau^{a} \otimes \gamma_{k} \leftrightarrow \tau^{a} \otimes \gamma_{k} \gamma_{5} \\ \tau^{a} \otimes \gamma_{4} \leftrightarrow \tau^{a} \otimes \gamma_{4} \gamma_{5} \\ \tau^{a} \otimes \gamma_{k} \gamma_{j} \leftrightarrow \tau^{a} \otimes \gamma_{k} \gamma_{j} \gamma_{5} \\ \tau^{a} \otimes \gamma_{k} \gamma_{4} \leftrightarrow \tau^{a} \otimes \gamma_{k} \gamma_{4} \gamma_{5} \\ \tau^{a} \otimes \mathbf{1} \leftrightarrow \mathbf{1}_{f} \otimes \mathbf{1} \\ \tau^{a} \otimes \gamma_{k} \gamma_{j} \leftrightarrow \mathbf{1}_{f} \otimes \gamma_{k} \gamma_{j} \\ \tau^{a} \otimes \gamma_{k} \gamma_{4} \leftrightarrow \mathbf{1}_{f} \otimes \gamma_{k} \gamma_{4} \\ \tau^{a} \otimes \gamma_{5} \leftrightarrow \mathbf{1}_{f} \otimes \gamma_{5} \\ \tau^{a} \otimes \gamma_{k} \gamma_{j} \gamma_{5} \leftrightarrow \mathbf{1}_{f} \otimes \gamma_{k} \gamma_{j} \gamma_{5} \\ \tau^{a} \otimes \gamma_{k} \gamma_{4} \gamma_{5} \leftrightarrow \mathbf{1}_{f} \otimes \gamma_{k} \gamma_{4} \gamma_{5} \\ \mathbf{1}_{f} \otimes \mathbf{1} \leftrightarrow \mathbf{1}_{f} \otimes \gamma_{5} \\ \mathbf{1}_{f} \otimes \gamma_{k} \gamma_{j} \leftrightarrow \mathbf{1}_{f} \otimes \gamma_{k} \gamma_{j} \gamma_{5} \\ \mathbf{1}_{f} \otimes \gamma_{k} \gamma_{4} \leftrightarrow \mathbf{1}_{f} \otimes \gamma_{k} \gamma_{4} \gamma_{5} \\$\hline\end{aligned}

$h$-equivalent meson propagators

$\begin{aligned} \tau^{a} \otimes \gamma_{k} & \leftrightarrow \mathbf{1}_{f} \otimes \gamma_{k} \\ \tau^{a} \otimes \gamma_{4} & \leftrightarrow \mathbf{1}_{f} \otimes \gamma_{4} \\ \tau^{a} \otimes \gamma_{k} \gamma_{5} & \leftrightarrow \mathbf{1}_{f} \otimes \gamma_{k} \gamma_{5} \\ \tau^{a} \otimes \gamma_{4} \gamma_{5} & \leftrightarrow \mathbf{1}_{f} \otimes \gamma_{4} \gamma_{5} \\ \tau^{a} \otimes \mathbf{1} & \leftrightarrow \tau^{a} \otimes \gamma_{4} \\ \tau^{a} \otimes \gamma_{k} & \leftrightarrow \tau^{a} \otimes \gamma_{k} \gamma_{4} \\ \tau^{a} \otimes \gamma_{5} & \leftrightarrow \tau^{a} \otimes \gamma_{4} \gamma_{5} \\ \tau^{a} \otimes \gamma_{k} \gamma_{j} & \leftrightarrow \tau^{a} \otimes \gamma_{k} \gamma_{j} \gamma_{4} \\ \tau^{a} \otimes \gamma_{k} \gamma_{5} & \leftrightarrow \tau^{a} \otimes \gamma_{k} \gamma_{4} \gamma_{5} \\ \mathbf{1}_{f} \otimes \gamma_{k} & \leftrightarrow \mathbf{1}_{f} \otimes \gamma_{k} \gamma_{5} \\ \mathbf{1}_{f} \otimes \gamma_{4} & \leftrightarrow \mathbf{1}_{f} \otimes \gamma_{4} \gamma_{5} \\ \mathbf{1}_{f} \otimes \mathbf{1} & \leftrightarrow \mathbf{1}_{f} \otimes \gamma_{4} \\ \mathbf{1}_{f} \otimes \gamma_{k} & \leftrightarrow \mathbf{1}_{f} \otimes \gamma_{k} \gamma_{4} \\ \mathbf{1}_{f} \otimes \gamma_{5} & \leftrightarrow \mathbf{1}_{f} \otimes \gamma_{4} \gamma_{5} \\ \mathbf{1}_{f} \otimes \gamma_{k} \gamma_{j} & \leftrightarrow \mathbf{1}_{f} \otimes \gamma_{k} \gamma_{j} \gamma_{4} \\ \mathbf{1}_{f} \otimes \gamma_{k} \gamma_{5} & \leftrightarrow \mathbf{1}_{f} \otimes \gamma_{k} \gamma_{4} \gamma_{5}\end{aligned}$

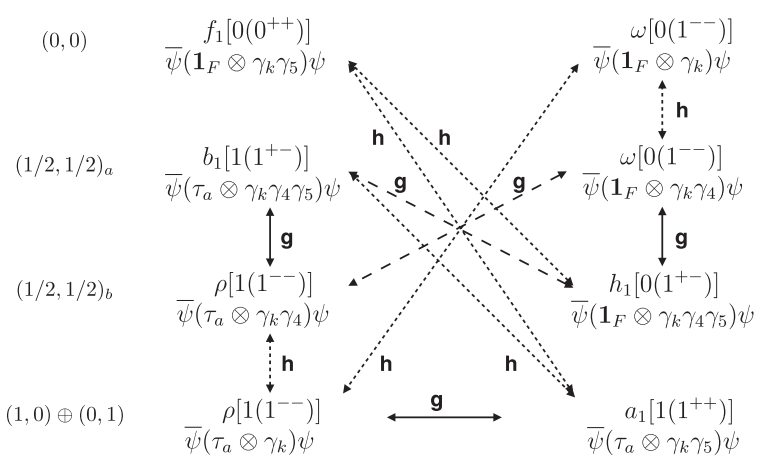

FIG. 2. The equivalence relations between the corresponding meson propagators for vector mesons are shown. The arrows symbolize the entries in Table II and the equivalence type as discussed in the text is shown. The arrangement of the operators follows [12] for better comparison; the left-hand column indicates the chiral structure [24].

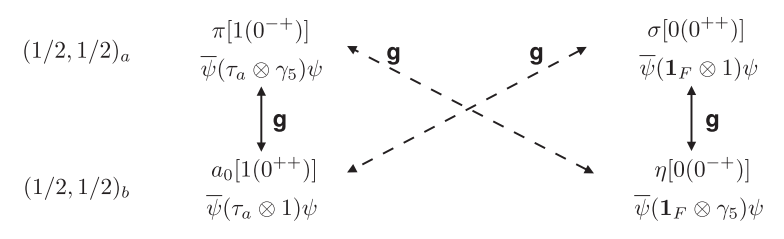

FIG. 3. The equivalence relations between the corresponding meson propagators for scalar mesons are shown. The $h$-equivalences have been omitted (although they are listed in Table II) as they relate to unphysical states, The left-hand column indicates the chiral structure [24].

$$
\begin{aligned}
P_{c}(\Gamma, x, y)-P_{c}\left(\mathrm{i} \Gamma \gamma_{5}, x, y\right) \\
=-8 \sum_{n>0, k>0} g\left(m, \eta_{n}\right) g\left(m, \eta_{k}\right)\left[\psi_{x, a}^{(k) \dagger} \Gamma_{a, b} \psi_{x, b}^{(n)} \psi_{y, c}^{(n) \dagger} \Gamma_{c, d} \psi_{y, d}^{(k)}\right. \\
\left.\quad+\psi_{x, a}^{(k) \dagger} \Gamma_{a, b} \psi_{x, b}^{(-n)} \psi_{y, c}^{(-n) \dagger} \Gamma_{c, d} \psi_{y, d}^{(k)}\right] .
\end{aligned}
$$

These propagator pairs are $g$-equivalent.

For small masses $m \rightarrow 0$ the functions $g$ emphasize the contributions of small eigenvalues. If the eigenvalue density $\rho(m, \eta)$ vanishes at small eigenvalues, then the propagator difference vanishes as well and axial symmetry is restored. The factors with eigenvectors are bounded (the eigenvectors are normalized).

The integral over $x, y$ and sum over $a$ and the other, hidden indices gives the susceptibility. For $\Gamma=\mathbf{1}$ the second term in (23) vanishes due to orthogonality. The first term gives $\delta_{n k}$. The susceptibility difference (the $U_{A}(1)$ susceptibility) then is

$$
\begin{aligned}
\chi(\mathbf{1})-\chi\left(\mathrm{i} \gamma_{5}\right) & =-\frac{4}{V} \sum_{n>0} g\left(m, \eta_{n}\right)^{2} \\
& \simeq \int_{0}^{\infty} d \eta \rho(m, \eta) g(m, \eta)^{2} .
\end{aligned}
$$

for the eigenvalue density $\rho(m, \eta)$ (cf. [15], the discussion in [17] and [8-11,16]). This term vanishes if there is a gap in $\rho(m, \eta)$ or if the density vanishes fast enough ${ }^{2}$ for $\eta \rightarrow 0$. The susceptibilities for the other difference pairs of Table I are also $g$-equivalent.

\section{Isovector vs isoscalar}

Isoscalar propagators have also disconnected contributions. For $\Gamma \in\left\{\mathbf{1}, \gamma_{k} \gamma_{j}, \gamma_{k} \gamma_{4}, \mathrm{i} \gamma_{5}, \mathrm{i} \gamma_{k} \gamma_{j} \gamma_{5}, \mathrm{i} \gamma_{k} \gamma_{4} \gamma_{5}\right\}$ they have the form (for the derivation see Appendix B)

$$
\begin{aligned}
& \sum_{n>0, k>0} 4 g\left(m, \eta_{n}\right) g\left(m, \eta_{k}\right) \\
& \quad \times\left(\psi^{(n) \dagger}(x) \Gamma_{\mathrm{snk}} \psi^{(n)}(x)\right)\left(\psi^{(k) \dagger}(y) \Gamma_{\mathrm{src}} \psi^{(k)}(y) .\right.
\end{aligned}
$$

We find that if the eigenvalue density at small eigenvalues vanishes, the disconnected term vanishes as well. The isoscalar is $g$-equivalent to the isovector propagators for

\footnotetext{
${ }^{2}$ A behavior $\lim _{m \rightarrow 0} \rho(m, \eta)=\mathcal{O}\left(\eta^{3}\right)$ is sufficient for the vanishing of the $U_{A}(1)$ susceptibility [16].
} 
these $\Gamma$. Combining the relations with those of Sec. III A 1 one obtains further relations between isoscalar pairs $\left(\mathbf{1}, \gamma_{5}\right),\left(\mathrm{i} \gamma_{k} \gamma_{j}, \mathrm{i} \gamma_{k} \gamma_{j} \gamma_{5}\right)$ and $\left(\gamma_{k} \gamma_{4}, \gamma_{k} \gamma_{4} \gamma_{5}\right)$. The scalar mesons at high temperature were studied in [6].

\section{B. $h$-equivalent mesons}

\section{More disconnected terms}

Compared with $g$-equivalence the now discussed type is more subtle with factors $h(m, \eta)$, needing further bounds or eigenmode properties to find propagator agreement. To see this we use the chiral basis of Sec. II B.

The disconnected contributions for propagators with $\Gamma \in$ $\left\{\gamma_{k}, \gamma_{4}, \gamma_{k} \gamma_{5}, \gamma_{4} \gamma_{5}\right\}$ have terms with factors like

$h\left(m, \eta_{n}\right) h\left(m, \eta_{k}\right) \times\left(R^{(n) \dagger}(x) \sigma L^{(n)}(x)\right)\left(L^{(k) \dagger}(y) \sigma R^{(k)}(y)\right)$,

where $\sigma$ is a $2 \times 2$ matrix (i.e., a sub-block of $\Gamma$; for the complete expression see Appendix C). The prefactor again favors low eigenmodes for small $\mathrm{m}$. Therefore the disconnected contributions become much smaller if the low modes are suppressed in the generic density.

Even if the low modes are absent, however, the higher modes still contribute to the difference more than in the $g$-equivalent case since $h(m, \eta)$ decreases slower with $\eta$ than $g(m, \eta)$. The quality of the agreement then depends on the matrix elements in (26) and not only on the eigenvalue density. This is discussed in the subsequent section.

If the high modes contribution can be neglected the isoscalar propagator agrees with the isovector propagator for the listed $\Gamma$. Considering the results for the connected propagators this implies also agreement of the isoscalar propagator pairs $\left(\gamma_{k}, \gamma_{k} \gamma_{5}\right)$ and $\left(\gamma_{4}, \gamma_{4} \gamma_{5}\right)$.

\section{2. $\Gamma v s \Gamma \gamma_{4}$}

Finally let us consider the connected propagator pairs for $\left(\Gamma, \Gamma \gamma_{4}\right)$ for $\Gamma \in\left\{\mathbf{1}, \gamma_{k}, \gamma_{5}, \gamma_{k} \gamma_{j}, \gamma_{k} \gamma_{5}\right\}$; these are central for the CS symmetry. The propagator differences are sums of two types of terms

$$
\begin{aligned}
& g\left(m, \eta_{n}\right) g\left(m, \eta_{k}\right)\left(\left(L^{(n) \dagger}(x) \sigma L^{(k)}(x)\right)\left(L^{(k) \dagger}(y) \sigma L^{(n)}(y)\right)+\left(R^{(n) \dagger}(x) \sigma R^{(k)}(x)\right)\left(R^{(k) \dagger}(y) \sigma R^{(n)}(y)\right)\right. \\
& \left.-\left(R^{(n) \dagger}(x) \sigma L^{(k)}(x)\right)\left(L^{(k) \dagger}(y) \sigma R^{(n)}(y)\right)-\left(L^{(n) \dagger}(x) \sigma R^{(k)}(x)\right)\left(R^{(k) \dagger}(y) \sigma L^{(n)}(y)\right)\right)
\end{aligned}
$$

and

$$
\begin{aligned}
& h\left(m, \eta_{n}\right) h\left(m, \eta_{k}\right)\left(\left(R^{(n) \dagger}(x) \sigma R^{(k)}(x)\right)\left(L^{(k) \dagger}(y) \sigma L^{(n)}(y)\right)+\left(L^{(n) \dagger}(x) \sigma L^{(k)}(x)\right)\left(R^{(k) \dagger}(y) \sigma R^{(n)}(y)\right)\right. \\
& \left.\quad-\left(L^{(n) \dagger}(x) \sigma R^{(k)}(x)\right)\left(L^{(k) \dagger}(y) \sigma R^{(n)}(y)\right)-\left(R^{(n) \dagger}(x) \sigma L^{(k)}(x)\right)\left(R^{(k) \dagger}(y) \sigma L^{(n)}(y)\right)\right)
\end{aligned}
$$

The first term becomes negligible if the fermion mass is small and if there is a gap in $\rho(m, \eta)$ at small $\eta$. In the second term, unlike the connected propagators discussed in Sec. III A 1, all four types $R^{\dagger} \sigma L, L^{\dagger} \sigma R, R^{\dagger} \sigma R, L^{\dagger} \sigma L$ enter the propagator difference multiplying $h$.

When there are no eigenvalues below some $|\eta|<\eta_{0}$ or the generic density vanishes fast enough toward $\eta=0$ the propagator difference is dominated by the terms with $h$. The factors $L^{\dagger} \sigma R$, etc., encode the dynamics of QCD.

There are a few observations that may shed some light:

(i) The $h$ - and $g$-terms of the difference pairs $\left(\mathbf{1}, \gamma_{4}\right)$ and $\left(\mathrm{i} \gamma_{5}, \mathrm{i} \gamma_{4} \gamma_{5}\right)$ are identical, as are those for $\left(\gamma_{k}, \gamma_{k} \gamma_{4}\right)$ and $\left(\mathrm{i} \gamma_{k} \gamma_{5}, \mathrm{i} \gamma_{k} \gamma_{4} \gamma_{5}\right)$. In other words, if the propagator for $\gamma_{k}$ and $\gamma_{k} \gamma_{4}$ agree, so do the propagators for $\mathrm{i} \gamma_{k} \gamma_{5}$ and $\mathrm{i} \gamma_{k} \gamma_{4} \gamma_{5}$.

(ii) The $h$-terms vanish for chiral eigenmodes of the form $(R, 0)$ or $(0, L)$ or will be suppressed for almost chiral eigenmodes (where either $|R| \gg|L|$ or $|R| \ll|L|$ ). However, such behavior is expected mainly for the low-lying modes which are truncated or suppressed anyhow in the situation of relevance here.

(iii) The mesons with $\Gamma=\gamma_{4}$ or $\gamma_{4} \gamma_{5}$ have only terms $R^{\dagger} L$ and $L^{\dagger} R$; now $R^{\dagger}$ and $L$ correspond to the same helicity which cannot add up to zero. The states cannot be physical scalars [12]. For this reason we omit these states in Fig. 3.

(iv) There is numerical evidence [4] indicating that the scalar propagators show less agreement than the vector propagators. This is a hint that the vector matrix elements $\psi^{(n) \dagger} \gamma_{j} \psi^{(k)}$ are smaller than the scalar ones $\psi^{(n) \dagger} \psi^{(k)}$.

\section{CONCLUSIONS}

Here we studied the role of low-lying eigenmodes of the Dirac operator in meson propagators. The study is motivated by lattice QCD calculations where it was found that the differences between meson propagators of a large class disappear if the low-lying (i.e., close to zero) modes of the Dirac operator are suppressed. The mass degeneracies have been observed when the low modes were truncated explicitly $[3-5,12]$ or dynamically suppressed at large temperature [7]. ${ }^{3}$

\footnotetext{
${ }^{3} \mathrm{~A}$ dominance of the low-lying modes for some meson propagators has been noticed earlier (see, e.g., [25]).
} 
There are two qualitatively different kinds of relations. Those with a weight factor $g(m, \eta)$ we call $g$-equivalent. Meson propagators that are $g$-equivalent (Figs. 2 and 3 and Table II) approach each other for small quark mass, if there is a low-eigenvalue suppression or gap in the generic eigenvalue density. These equivalences, when realized, restore the axial symmetries $S U(2)_{A}$ and $U(1)_{A}$.

The second type called $h$-equivalence needs further constraints in order to provide vanishing propagator differences. The weight factor $h(m, \eta)$ is also peaked at small $\eta$ but does not suppress the higher modes as efficient. In that case the quality of agreement depends on the overlap of eigenvectors.

We find:

(i) The connected (isovector) propagators $P_{c}(\Gamma)$ and $P_{c}\left(\mathrm{i} \Gamma \gamma_{5}\right)$ for $\Gamma \in\left\{\mathbf{1}, \gamma_{k}, \gamma_{4}, \gamma_{k} \gamma_{j}, \gamma_{k} \gamma_{4}\right\}$ differ only by $g$-type terms. If there is a low mode suppression the propagators of a pair agree with each other for $m \rightarrow 0$. The susceptibilities of the connected (isovector) propagators $P_{c}(\Gamma)$ inherit the $g$-equivalence property.

(ii) For some isoscalar mesons (see Sec. III A 2) the propagators' disconnected contributions are $g$-type terms. For these mesons the isoscalar and isovector propagators agree in the massless limit if there is a suppression of low eigenvalues.

(iii) The connected (isovector) propagators $P_{c}(\Gamma)$ and $P_{c}\left(\Gamma \gamma_{4}\right)$ for $\Gamma \in\left\{\mathbf{1}, \gamma_{k}, \gamma_{5}, \gamma_{k} \gamma_{j}, \gamma_{k} \gamma_{5}\right\}$. differ by $g$-type and $h$-type terms. The $h$-terms become small for almost chiral eigenmodes (where either $|R| \gg$ $|L|$ or $|R| \ll|L|)$ or small overlap $\phi^{(n) \dagger} \Gamma \phi^{(k)}$.

(iv) The propagator difference $\left(P_{c}\left(\gamma_{k}\right)-P_{c}\left(\gamma_{k} \gamma_{4}\right)\right)$ differs from $\left(P_{c}\left(\mathrm{i} \gamma_{k} \gamma_{5}\right)-P_{c}\left(\mathrm{i} \gamma_{k} \gamma_{4} \gamma_{5}\right)\right)$ only by $g$-type terms. That is, if the $h$-type contribution vanishes for one pair it also vanishes for the other. Also the propagator difference $\left(P_{c}(\mathbf{1})-P_{c}\left(\gamma_{4}\right)\right)$ differs from $\left(P_{c}\left(\mathrm{i} \gamma_{5}\right)-P_{c}\left(\mathrm{i} \gamma_{4} \gamma_{5}\right)\right)$ only by $g$-type terms.

In summary the axial symmetries between the meson propagators and susceptibilities are recovered for decreasing quark mass upon suppression of low-lying eigenmodes in the eigenvalue distribution. A similar behaviour for the observed $\gamma_{4}$ symmetry requires in addition small overlap of the higher eigenmodes.

The emerging agreement between the meson propagators explains numerical lattice QCD results for meson mass degeneracies. Based on the meson mass pattern the symmetries CS and $S U(4)$ were conjectured [12]. These may have far-reaching consequences [22].

\section{ACKNOWLEDGMENTS}

I profited much from discussions with Christof Gattringer and Vasily Sazonov. Many thanks go to Leonid Glozman for numerous discussions, for reading the manuscript, and for his persistence.

\section{APPENDIX A: $\boldsymbol{P}_{c}(\Gamma, x, y)-\boldsymbol{P}_{c}\left(\Gamma \gamma_{5}, x, y\right)$}

The connected propagator $P_{c}(\Gamma)$ for $\Gamma$ is written in terms of the spectral representation of the quark propagators:

$$
P_{c}(\Gamma)=s_{\Gamma} \sum_{n, k} f_{n} f_{k} \psi_{x, a}^{(k) \dagger} \Gamma_{a, b} \psi_{x, b}^{(n)} \psi_{y, c}^{(n) \dagger} \Gamma_{c, d} \psi_{y, d}^{(k)},
$$

with $s_{\Gamma}$ defined in Sec. III and Table I and summation over paired indices is implied. We use the abbreviation

$$
f_{n} \equiv \frac{1}{\eta_{n}+\mathrm{i} m}
$$

with $\eta_{-n}=-\eta_{n}$. There are no exact zero modes by assumption. We rewrite the sum like

$$
\begin{aligned}
& s_{\Gamma} \sum_{n>0, k>0}\left[f_{n} f_{k} \psi_{x, a}^{(k) \dagger} \Gamma_{a, b} \psi_{x, b}^{(n)} \psi_{y, c}^{(n) \dagger} \Gamma_{c, d} \psi_{y, d}^{(k)}+f_{-n} f_{-k} \psi_{x, a}^{(-k) \dagger} \Gamma_{a, b} \psi_{x, b}^{(-n)} \psi_{y, c}^{(-n) \dagger} \Gamma_{c, d} \psi_{y, d}^{(-k)}+f_{-n} f_{k} \psi_{x, a}^{(k) \dagger} \Gamma_{a, b} \psi_{x, b}^{(-n)} \psi_{y, c}^{(-n) \dagger} \Gamma_{c, d} \psi_{y, d}^{(k)}\right. \\
&\left.+f_{n} f_{-k} \psi_{x, a}^{(-k) \dagger} \Gamma_{a, b} \psi_{x, b}^{(n)} \psi_{y, c}^{(n) \dagger} \Gamma_{c, d} \psi_{y, d}^{(-k)}\right] \\
&= s_{\Gamma} \sum_{n>0, k>0}\left[f_{n} f_{k} \psi_{x, a}^{(k) \dagger} \Gamma_{a, b} \psi_{x, b}^{(n)} \psi_{y, c}^{(n) \dagger} \Gamma_{c, d} \psi_{y, d}^{(k)}+f_{-n} f_{-k} \psi_{x, a}^{(k) \dagger} \Gamma_{a, b} \psi_{x, b}^{(n)} \psi_{y, c}^{(n) \dagger} \Gamma_{c, d} \psi_{y, d}^{(k)}+f_{-n} f_{k} \psi_{x, a}^{(k) \dagger} \Gamma_{a, b} \psi_{x, b}^{(-n)} \psi_{y, c}^{(-n) \dagger} \Gamma_{c, d} \psi_{y, d}^{(k)}\right. \\
&\left.+f_{n} f_{-k} \psi_{x, a}^{(k) \dagger} \Gamma_{a, b} \psi_{x, b}^{(-n)} \psi_{y, c}^{(-n) \dagger} \Gamma_{c, d} \psi_{y, d}^{(k)}\right] \\
&= s_{\Gamma} \sum_{n>0, k>0}\left[\left(f_{n} f_{k}+f_{-n} f_{-k}\right) \psi_{x, a}^{(k) \dagger} \Gamma_{a, b} \psi_{x, b}^{(n)} \psi_{y, c}^{(n) \dagger} \Gamma_{c, d} \psi_{y, d}^{(k)}+\left(f_{-n} f_{k}+f_{n} f_{-k}\right) \psi_{x, a}^{(k) \dagger} \Gamma_{a, b} \psi_{x, b}^{(-n)} \psi_{y, c}^{(-n) \dagger} \Gamma_{c, d} \psi_{y, d}^{(k)}\right] \\
&= s_{\Gamma} \sum_{n>0, k>0}\left[\left(-2 g\left(m, \eta_{n}\right) g\left(m, \eta_{k}\right)+2 h\left(m, \eta_{n}\right) h\left(m, \eta_{k}\right)\right) \psi_{x, a}^{(k) \dagger} \Gamma_{a, b} \psi_{x, b}^{(n)} \psi_{y, c}^{(n) \dagger} \Gamma_{c, d} \psi_{y, d}^{(k)}\right. \\
&\left.+\left(-2 g\left(m, \eta_{n}\right) g\left(m, \eta_{k}\right)-2 h\left(m, \eta_{n}\right) h\left(m, \eta_{k}\right)\right) \psi_{x, a}^{(k) \dagger} \Gamma_{a, b} \psi_{x, b}^{(-n)} \psi_{y, c}^{(-n) \dagger} \Gamma_{c, d} \psi_{y, d}^{(k)}\right]
\end{aligned}
$$

Here we used relations like (20), (22) and 


$$
\begin{aligned}
& f_{n} f_{k}+f_{-n} f_{-k}=-2 g\left(m, \eta_{n}\right) g\left(m, \eta_{k}\right)+2 h\left(m, \eta_{n}\right) h\left(m, \eta_{k}\right) \\
& f_{-n} f_{k}+f_{n} f_{-k}=-2 g\left(m, \eta_{n}\right) g\left(m, \eta_{k}\right)-2 h\left(m, \eta_{n}\right) h\left(m, \eta_{k}\right) .
\end{aligned}
$$

The propagator is

$$
\begin{aligned}
P_{c}\left(\mathrm{i} \Gamma \gamma_{5}\right)= & \mathrm{i}^{2} s_{\Gamma \gamma 5} \sum_{n>0, k>0}\left[\left(-2 g\left(m, \eta_{n}\right) g\left(m, \eta_{k}\right)+2 h\left(m, \eta_{n}\right) h\left(m, \eta_{k}\right)\right) \psi_{x, a}^{(k) \dagger}\left(\Gamma \gamma_{5}\right)_{a, b} \psi_{x, b}^{(n)} \psi_{y, c}^{(n) \dagger}\left(\Gamma \gamma_{5}\right)_{c, d} \psi_{y, d}^{(k)}\right. \\
& \left.+\left(-2 g\left(m, \eta_{n}\right) g\left(m, \eta_{k}\right)-2 h\left(m, \eta_{n}\right) h\left(m, \eta_{k}\right)\right) \psi_{x, a}^{(k) \dagger}\left(\Gamma \gamma_{5}\right)_{a, b} \psi_{x, b}^{(-n)} \psi_{y, c}^{(-n) \dagger}\left(\Gamma \gamma_{5}\right)_{c, d} \psi_{y, d}^{(k)}\right] \\
= & -s_{\Gamma \gamma 5} s_{5} \sum_{n>0, k>0}\left[\left(-2 g\left(m, \eta_{n}\right) g\left(m, \eta_{k}\right)+2 h\left(m, \eta_{n}\right) h\left(m, \eta_{k}\right)\right) \psi_{x, a}^{(k) \dagger} \Gamma_{a, b} \psi_{x, b}^{(-n)} \psi_{y, c}^{(-n) \dagger} \Gamma_{c, d} \psi_{y, d}^{(k)}\right. \\
& \left.+\left(-2 g\left(m, \eta_{n}\right) g\left(m, \eta_{k}\right)-2 h\left(m, \eta_{n}\right) h\left(m, \eta_{k}\right)\right) \psi_{x, a}^{(k) \dagger} \Gamma_{a, b} \psi_{x, b}^{(n)} \psi_{y, c}^{(n) \dagger} \Gamma_{c, d} \psi_{y, d}^{(k)}\right]
\end{aligned}
$$

where we replaced in (A3) $s_{\Gamma}$ by $s_{\Gamma \gamma 5}$ and $\Gamma$ by $\Gamma \gamma_{5}$ and utilized (20).

The difference between the propagators becomes in all cases

$$
P_{c}(\Gamma)-P_{c}\left(\Gamma \gamma_{5}\right)=-4 \sum_{n>0, k>0} g\left(m, \eta_{n}\right) g\left(m, \eta_{k}\right)\left[\psi_{x, a}^{(k) \dagger} \Gamma_{a, b} \psi_{x, b}^{(n)} \psi_{y, c}^{(n) \dagger} \Gamma_{c, d} \psi_{y, d}^{(k)}+\psi_{x, a}^{(k) \dagger} \Gamma_{a, b} \psi_{x, b}^{(-n)} \psi_{y, c}^{(-n) \dagger} \Gamma_{c, d} \psi_{y, d}^{(k)}\right]
$$

\section{APPENDIX B: DISCONNECTED TERMS}

These terms are responsible for the difference between isovector and isoscalar propagators and have the form

$P_{d}(\Gamma)=-s_{\Gamma}\left[\sum_{k} f_{k} \psi_{x, a}^{(k) \dagger} \Gamma_{a, b} \psi_{x, b}^{(k)}\right]\left[\sum_{n} f_{n} \psi_{y, c}^{(n) \dagger} \Gamma_{c, d} \psi_{y, d}^{(n)}\right]$.

Rewriting the first sum gives

$$
\begin{aligned}
& -\sum_{k>0}\left[f_{k} \psi_{x, a}^{(k) \dagger} \Gamma_{a, b} \psi_{x, b}^{(k)}+f_{-k} \psi_{x, a}^{(-k) \dagger} \Gamma_{a, b} \psi_{x, b}^{(-k)}\right] \\
& =\sum_{k>0}\left[f_{k} \psi_{x, a}^{(k) \dagger} \Gamma_{a, b} \psi_{x, b}^{(k)}+f_{-k} s_{5} \psi_{x, a}^{(k) \dagger} \Gamma_{a, b} \psi_{x, b}^{(k)}\right] \\
& =-\sum_{k>0}\left(f_{k}+s_{5} f_{-k}\right) \psi_{x, a}^{(k) \dagger} \Gamma_{a, b} \psi_{x, b}^{(k)}
\end{aligned}
$$

where we used (20) in the 2nd step. Equivalent derivation for the 2 nd sum leads to

$$
\begin{aligned}
P_{d}(\Gamma)= & -s_{\Gamma} \sum_{k>0 . n>0}\left(f_{k}+s_{5} f_{-k}\right)\left(f_{n}+s_{5} f_{-n}\right) \\
& \times \psi_{x, a}^{(k) \dagger} \Gamma_{a, b} \psi_{x, b}^{(k)} \psi_{y, c}^{(n) \dagger} \Gamma_{c, d} \psi_{y, d}^{(n)}
\end{aligned}
$$

For $\Gamma \in\left\{\mathbf{1}, \gamma_{k} \gamma_{j}, \gamma_{k} \gamma_{4}, \mathrm{i} \gamma_{5}, \mathrm{i} \gamma_{k} \gamma_{j} \gamma_{5}, \mathrm{i} \gamma_{k} \gamma_{4} \gamma_{5}\right\}$ we find $s_{5}=1$ and

$$
\left(f_{k}+f_{-k}\right)\left(f_{n}+f_{-n}\right)=-4 g\left(m, \eta_{k}\right) g\left(m, \eta_{n}\right)
$$

giving (25).
For $\Gamma \in\left\{\gamma_{k}, \gamma_{4}, \gamma_{k} \gamma_{5}, \gamma_{4} \gamma_{5}\right\}$ we find $s_{5}=-1$ and

$$
\left(f_{k}-f_{-k}\right)\left(f_{n}-f_{-n}\right)=4 h\left(m, \eta_{k}\right) h\left(m, \eta_{n}\right)
$$

which is discussed below in Appendix C.

\section{APPENDIX C: MORE DISCONNECTED TERMS}

This concerns the disconnected terms (B5) in Sec. III B 1. We consider the disconnected contribution to propagators for $\Gamma \in\left\{\gamma_{k}, \gamma_{4}, \gamma_{k} \gamma_{5}, \gamma_{4} \gamma_{5}\right\}$ discussed at the end of Appendix B. Since the functions $h(m, \eta)$ have slower decay towards larger $\eta$ we have a closer look at the matrix elements. In the chiral basis of (13) the matrices $\Gamma$ have the form

$$
\left(\begin{array}{ll}
0 & \sigma \\
\sigma & 0
\end{array}\right) \text { or }\left(\begin{array}{cc}
0 & -\sigma \\
\sigma & 0
\end{array}\right)
$$

Then in all cases we find the form ( $\sigma$ depends on the actual $\Gamma$ and is proportional to a Pauli matrix)

$$
\begin{aligned}
P_{d}(\Gamma)= & 4 s_{\Gamma} \sum_{k>0 . n>0} h\left(m, \eta_{k}\right) h\left(m, \eta_{n}\right) \\
& \times\left(R^{(n) \dagger}(x) \sigma L^{(n)}(x)+L^{(n) \dagger}(x) \sigma R^{(n)}(x)\right) \\
& \times\left(R^{(k) \dagger}(y) \sigma L^{(k)}(y)+L^{(k) \dagger}(y) \sigma R^{(k)}(y)\right),
\end{aligned}
$$

In all terms upper components couple to lower ones. If the overlap is small (e.g., is the eigenmodes are close to chiral) then this contribution is small and the isovector and isoscalar propagators for that $\Gamma$ are similar. 


\section{APPENDIX D: $\boldsymbol{P}_{c}(\Gamma)-\boldsymbol{P}_{c}\left(\Gamma \gamma_{4}\right)$}

Also the connected propagator differences between these pairs need additional assumptions like those of Appendix C. We inspect pairs $\left(\Gamma, \Gamma \gamma_{4}\right)$ for $\Gamma \in\left\{\mathbf{1}, \gamma_{k}, \gamma_{5}, \gamma_{k} \gamma_{j}, \gamma_{k} \gamma_{5}\right\}$

Using (A3) we get

$$
\begin{aligned}
P_{c}(\Gamma)-P_{c}\left(\Gamma \gamma_{4}\right)= & \sum_{n>0, k>0}\left[( - 2 g ( m , \eta _ { n } ) g ( m , \eta _ { k } ) + 2 h ( m , \eta _ { n } ) h ( m , \eta _ { k } ) ) \left(s_{\Gamma} \psi_{x, a}^{(k) \dagger} \Gamma_{a, b} \psi_{x, b}^{(n)} \psi_{y, c}^{(n) \dagger} \Gamma_{c, d} \psi_{y, d}^{(k)}\right.\right. \\
& \left.-s_{\Gamma \gamma_{4}} \psi_{x, a}^{(k) \dagger}\left(\Gamma \gamma_{4}\right)_{a, b} \psi_{x, b}^{(n)} \psi_{y, c}^{(n) \dagger}\left(\Gamma \gamma_{4}\right)_{c, d} \psi_{y, d}^{(k)}\right) \\
& +\left(-2 g\left(m, \eta_{n}\right) g\left(m, \eta_{k}\right)-2 h\left(m, \eta_{n}\right) h\left(m, \eta_{k}\right)\right)\left(s_{\Gamma} \psi_{x, a}^{(k) \dagger} \Gamma_{a, b} \psi_{x, b}^{(-n)} \psi_{y, c}^{(-n) \dagger} \Gamma_{c, d} \psi_{y, d}^{(k)}\right. \\
& \left.\left.\left.-s_{\Gamma \gamma_{4}} \psi_{x, a}^{(k) \dagger}\left(\Gamma \gamma_{4}\right)_{a, b} \psi_{x, b}^{(-n)} \psi_{y, c}^{(-n) \dagger}\left(\Gamma \gamma_{4}\right)_{c, d} \psi_{y, d}^{(k)}\right)\right)\right]
\end{aligned}
$$

We change to formulation (13); the matrix pair $\Gamma$ and $\Gamma \gamma_{4}$ have a form like, e.g.,

$$
\left(\begin{array}{ll}
\sigma & 0 \\
0 & \sigma
\end{array}\right) \text { and }\left(\begin{array}{ll}
0 & \sigma \\
\sigma & 0
\end{array}\right)
$$

As example we take $\Gamma=\mathbf{1}$ and $\Gamma \gamma_{4}=\gamma_{4}$ where $s_{\Gamma}=s_{\Gamma \gamma_{4}}=1$. (The other combinations give similar results, differing only in some signs.) Then (D1) becomes

$$
\begin{aligned}
4 & \sum_{n>0, k>0}\left[g ( m , \eta _ { k } ) g ( m , \eta _ { n } ) \left(-L^{(n) \dagger}(x) L^{(k)}(x) L^{(k) \dagger}(y) L^{(n)}(y)+R^{(n) \dagger}(x) L^{(k)}(x) L^{(k) \dagger}(y) R^{(n)}(y)\right.\right. \\
& \left.+L^{(n) \dagger}(x) R^{(k)}(x) R^{(k) \dagger}(y) L^{(n)}(y)-R^{(n) \dagger}(x) R^{(k)}(x) R^{(k) \dagger}(y) R^{(n)}(y)\right)+h\left(m, \eta_{k}\right) h\left(m, \eta_{n}\right)\left(-L^{(n) \dagger}(x) R^{(k)}(x) L^{(k) \dagger}(y) R^{(n)}(y)\right. \\
& \left.\left.-R^{(n) \dagger}(x) L^{(k)}(x) R^{(k) \dagger}(y) L^{(n)}(y)+R^{(n) \dagger}(x) R^{(k)}(x) L^{(k) \dagger}(y) L^{(n)}(y)+L^{(n) \dagger}(x) L^{(k)}(x) R^{(k) \dagger}(y) R^{(n)}(y)\right)\right] .
\end{aligned}
$$

Again we find a term with $g\left(m, \eta_{k}\right) g\left(m, \eta_{n}\right)$ which vanishes if the small modes disappear. The term with $h\left(m, \eta_{k}\right) h\left(m, \eta_{n}\right)$ has significant contributions from low modes which disappear with them. It decays, however, slower for increasing $\eta$. If the modes above the gap are close to chiral, this term becomes small as well. In that case we are left with the $g$-type terms and the propagators are $g$-equivalent.

[1] C. B. Lang and M. Schröck, Phys. Rev. D 84, 087704 (2011).

[2] L. Y. Glozman, C. B. Lang, and M. Schröck, Phys. Rev. D 86, 014507 (2012).

[3] M. Denissenya, L. Y. Glozman, and C. B. Lang, Phys. Rev. D 89, 077502 (2014).

[4] M. Denissenya, L. Y. Glozman, and C. B. Lang, Phys. Rev. D 91, 034505 (2015).

[5] M. Denissenya, L. Y. Glozman, and M. Pak, Phys. Rev. D 91, 114512 (2015).

[6] A. Tomiya, G. Cossu, S. Aoki, H. Fukaya, S. Hashimoto, T. Kaneko, and J. Noaki, Phys. Rev. D 96, 034509 (2017).

[7] C. Rohrhofer, Y. Aoki, G. Cossu, H. Fukaya, L. Ya. Glozman, S. Hashimoto, C. B. Lang, and S. Prelovsek, Phys. Rev. D 96, 094501 (2017).

[8] V. Dick, F. Karsch, E. Laermann, S. Mukherjee, and S. Sharma, Phys. Rev. D 91, 094504 (2015).
[9] A. Tomiya, G. Cossu, H. Fukaya, S. Hashimoto, and J. Noaki, Proc. Sci., LATTICE2014 (2015) 211 [arXiv: 1412.7306].

[10] H. Ohno, U. M. Heller, F. Karsch, and S. Mukherjee, Proc. Sci., LATTICE2012 (2012) 095 [arXiv:1211.2591].

[11] T. G. Kovacs, Proc. Sci., LATTICE2008 (2008) 198 [arXiv:0810.4763].

[12] L. Y. Glozman and M. Pak, Phys. Rev. D 92, 016001 (2015).

[13] L. Y. Glozman, Eur. Phys. J. A 51, 27 (2015).

[14] T. Banks and A. Casher, Nucl. Phys. B169, 103 (1980).

[15] T. D. Cohen, Phys. Rev. D 54, R1867 (1996).

[16] S. Aoki, H. Fukaya, and Y. Taniguchi, Phys. Rev. D 86, 114512 (2012).

[17] H. Fukaya, EPJ Web Conf. 175, 01012 (2018).

[18] K. Fujikawa, Phys. Rev. Lett. 42, 1195 (1979).

[19] K. Fujikawa, Phys. Rev. D 21, 2848 (1980); 22, 1499(E) (1980). 
[20] K. Fujikawa and H. Suzuki, Path Integrals and Quantum Anomalies (Claredon Press, Oxford, 2004).

[21] M. Denissenya, L. Y. Glozman, and M. Pak, Phys. Rev. D 92, 074508 (2015).

[22] L. Y. Glozman, arXiv:1712.05168.
[23] J. J. M. Verbaarschot, NATO Sci. Ser. B 368, 343 (1998).

[24] T. D. Cohen and X.-D. Ji, Phys. Rev. D 55, 6870 (1997).

[25] T. A. DeGrand and A. Hasenfratz, Phys. Rev. D 64, 034512 (2001). 